Background: Articular involvement in acute gout attack is extremely common and mainly characterized by arthritis, which are usually transient, severe, reversible and well responsive to treatment. The involvement of tendons and entheses in lower extremity in monosodium urate-related disease through US (ultrasound) assessment have been described ${ }^{1,2}$. US findings in gout raising the hypothesis that entheseal involvement could be a missing target in the clinical evaluation of gout patients.

Objectives: To evaluate by ultrasound (US) the frequency and characteristics of lower extremity entheseal involvement in acute gout attack patients.

Methods: US assessment were performed by independent rheumatologist on 31 patients with acute gout attack. Presence of lower extremity entheseal involvement were evaluated by grey-scale (GS) and power Doppler (PD). US assessment contain quadriceps, patellar and Achilles tendons, and plantar fascia entheses according to the OMERACT definitions

Results: US revealed one or more abnormalities in at least one enthesis in 22 out of 31 gout patients $(71.0 \%)$ and 47 out of 310 entheses $(15.2 \%)$. Among the affected entheses, the patellar insertion of quadriceps tendon was most commonly involved $(57.4 \%)$ during acute gout attack, followed by the calcaneal insertion of the Achilles tendon (17.0\%) and distal insertion of the patellar tendon (14.9\%). The proximal insertion of the patellar tendon and calcaneal insertion of the plantar fascia were involved in $8.5 \%$ and $2.1 \%$, respectively. Bone erosions and osteophytes were found in affected entheses ( $10.6 \%$ and $25.5 \%$, respectively).

Conclusion: Our study identifies that lower extremity entheseal involvement is a missing target in the evaluation of patients with acute gout attack. US plays a key role in the assessment of both clinical and subclinical enthesitis in gout patients. References:

[1] Pineda C, Amezcua-Guerra LM, Solano C, et al. Joint and Tendon Subclinical Involvement Suggestive of Gouty Arthritis in Asymptomatic Hyperuricemia: an Ultrasound Controlled Study. J Rheumatol. 2011;38(6):1195-1195.

[2] Carroll M, Dalbeth N, Allen B, et al. Ultrasound Characteristics of the Achilles Tendon in Tophaceous Gout: A Comparison with Age- and Sex-matched Controls. J Rheumatol. 2017;44(10):1487-1492.

Disclosure of Interests: None declared

DOI: 10.1136/annrheumdis-2020-eular.4138

\section{AB0920 \\ APPLICATION OF THE EULAR 2016 GUIDELINES FOR URATE-LOWERING THERAPY IN CLINICAL PRACTICE (DATA OF A SIX-MONTH PROSPECTIVE STUDY)}

M. Chikina ${ }^{1}$, M. Eliseev ${ }^{1}$, O. Sheliabina ${ }^{1}{ }^{1}$ V.A. Nasonova Research Institute of Rheumatology, V.A. Nasonova Research Institute of Rheumatology, Moscow, Russian Federation

Background: The EULAR 2016 guidelines on gout management provide for a consecutive regimen of urate-lowering medications; however, the possibility of reaching the target uric acid level when using the regimen has not been studied in clinical practice.

Objectives: To assess the possibility of reaching the target uric acid level when following the EULAR 2016 guidelines on gout management with use of different xanthine oxidase inhibitors available in Russia.

Methods: This monocentric prospective study included 83 gout patients (79 $(95 \%)$ male and $4(5 \%)$ female patients) with the mean age of $51.3 \pm 10.9$ years old. The inclusion criterion was indications for urate-lowering therapy in accordance with the EULAR 2016 guidelines. The exclusion criteria were the following: absolute contraindications to all of the study drugs, GFR $<30 \mathrm{ml} / \mathrm{min} / 1.73 \mathrm{~m} 2$, and NYHA class III-IV heart failure.

At urate-lowering therapy initiation, the patients were prescribed allopurinol with the starting dose of $100 \mathrm{mg} /$ day; the dose was titrated until the target uric acid level was reached (maximum up to $900 \mathrm{mg} /$ day), and in the patients with GFR between 30 and $60 \mathrm{ml} / \mathrm{min} / 1.73 \mathrm{~m} 2$ - up to $300 \mathrm{mg} /$ day. In case of insufficient efficacy of allopurinol (unachieved target uric acid level of $<360 \mu \mathrm{mol} / \mathrm{L}$, for the patients with severe gout of $<300 \mu \mathrm{mol} / \mathrm{L}$ ) or development of adverse reactions, allopurinol was replaced with febuxostat with the starting dose of $80 \mathrm{mg} /$ day and dose titration up to $120 \mathrm{mg} /$ day if necessary. The laboratory tests included serum creatinine level, uric acid level, AST, ALT, creatine phosphokinase, glucose; clinical blood test. The following parameters were assessed: possibility of reaching the target uric acid level when following the suggested regimen, and frequency of development of adverse reactions when using allopurinol and febuxostat.

Results: $37(45 \%)$ patients had the target uric acid level of $<360 \mu \mathrm{mol} / \mathrm{L}$ and $46(55 \%)$ pts - of $<300 \mu \mathrm{mol} / \mathrm{L}$. The recommended therapy regimen allowed 77 $(93 \%)$ patients under study to reach their target uric acid level.

The target uric acid level was achieved by $44(53 \%)$ out of 79 patients on allopurinol, of whom $36(82 \%)$ received $100-600 \mathrm{mg} /$ day, and $8(18 \%)-700-900 \mathrm{mg} /$ day. Of the patients with GFR of $>60 \mathrm{ml} / \mathrm{min} / 1.73 \mathrm{~m} 2,32(73 \%)$ patients achieved their target uric acid level and of those with GFR $<60 \mathrm{ml} / \mathrm{min} / 1.73 \mathrm{~m} 2$ - so did 12 (27\%). Then the patients on allopurinol with unachieved target uric acid level were prescribed febuxostat (in $30(77 \%)$ cases because of inefficacy of the allopurinol therapy, in $9(33 \%)$ patients because of their development of adverse reactions where 5 pts had a more than doubled level of transaminase (ALT, AST), 2 pts had skin itch and 2 pts had hives)

In total, 39 patients received febuxostat, of whom 4 pts were with initial intoler ance for allopurinol in past history, and 35 pts after therapy with maximum dose of allopurinol. A febuxostat dose of $80 \mathrm{mg} /$ day was associated with achievement of the target uric acid level in $14(42 \%)$ patients and that of $120 \mathrm{mg} /$ day - in $19(58 \%)$ patients, therefore, total $33(85 \%)$ patients reached their target uric acid level. Four patients on febuxostat developed adverse reactions: 3 patients had a more than doubled serum transaminase level (ALT, AST) and 1 patient had hives. Also, an insignificant increase in the mean GFR was registered from $73 \pm 21.4 \mathrm{ml} / \mathrm{min} / 1.73 \mathrm{~m} 2$ to $78.4 \pm 22.5 \mathrm{ml} / \mathrm{min} / 1.73 \mathrm{~m} 2$ which did not differ between the two drugs.

Conclusion: The recommended regimen of xanthine oxidase inhibitors in their maximal doses secures reaching the target uric acid level in $93 \%$ patients. In $47 \%$ patients, allopurinol in maximal doses (up to $900 \mathrm{mg} /$ day) does not significantly increase the possibility of reaching the target uric acid level, even though it demonstrates high tolerance.

Disclosure of Interests: Maria Chikina: None declared, Maxim Eliseev Speakers bureau: Novartis, Menarini Group, Alium, Olga Sheliabina: None declared DOI: 10.1136/annrheumdis-2020-eular.5171

\begin{tabular}{|l|l|}
\hline AB0921 & COMPARISON OF EFFICACY AND SAFETY OF \\
& DIFFERENT ANTI-INFLAMMATORY DRUGS AT \\
& INITIATION OF URATE-LOWERING THERAPY IN \\
& PATIENTS WITH GOUT (PRELIMINARY DATA)
\end{tabular}

M. Chikina ${ }^{1}$, M. Eliseev ${ }^{1}{ }^{1}$ V.A. Nasonova Research Institute of Rheumatology, V.A. Nasonova Research Institute of Rheumatology, Moscow, Russian Federation

Background: NSAIDs, colchicine and glucocorticoids are used for prevention of acute arthritis in gout patients, yet there is little data on their comparative efficacy. Objectives: Comparison of efficacy and safety of different anti-inflammatory drugs used for prevention of acute arthritis at initiation of urate-lowering therapy in gout patients.

Methods: This monocentric prospective study included 79 gout patients (75 $(94.9 \%)$ male and $4(5.1 \%)$ female patients) with the mean age of $51.3 \pm 10.9$ years old. The inclusion criteria were the following: established gout (ACR/ EULAR 2015 criteria), aged 18-80, serum uric acid level $>360 \mu \mathrm{mol} / \mathrm{L}$, absence of urate-lowering therapy at baseline and at least one gout flare within past three months. The exclusion criteria were: absolute contraindications to all of the study drugs, GFR $<30 \mathrm{ml} / \mathrm{min} / 1.73 \mathrm{~m} 2$.

All of the patients were prescribed urate-lowering therapy (allopurinol or febuxostat), the dose was titrated until the target serum uric-acid level $(<360 \mu \mathrm{mol} / \mathrm{L})$ was achieved. Simultaneously, preventive anti-inflammatory therapy was initiated and the drug for each patient was chosen individually: colchicine $0.5 \mathrm{mg} / \mathrm{day}$ or any NSAID in minimal anti-inflammatory dose or prednisolone $7.5 \mathrm{mg} / \mathrm{day}$. The analysis of the data included 3-month comparative evaluation of the efficacy of the preventive therapy against the following parameters: frequency of gout flare and duration thereof, VAS pain intensity of flare. The laboratory tests included serum creatinine level, uric acid level, AST, ALT, creatine phosphokinase, glucose; clinical blood test before, two weeks and three months after the initiation of the therapy.

Results: NSAIDs were received by 14 (17.7\%) patients, colchicine by $56(70.9 \%)$ and glucocorticoids by $9(11.4 \%)$ patients. There were no differences initially in age, GFR or lab test values.

Three months later, the gout flares frequency median lowered to $1[0 ; 2]$ flare $(p<0.01)$. The frequency of gout flares did not depend on the chosen drug and was $1[0 ; 1]$ for NSAIDs, $1[0 ; 2]$ for colchicine and 1 [1;2] for glucocorticoids. $40(50.6 \%)$ patients out of 79 did not have a single flare. The patients who received NSAIDs (57.1\%) and colchicine (42.9\%) experienced no gout flares more often than those who received glucocorticoids $(37.5 \%)$, but the differences were not significant.

However, the VAS pain intensity of gout flares in the patients who received NSAIDs $(30.7 \pm 12.9 \mathrm{~mm})$ was lower than in those who received colchicine $(42.1 \pm 12.3 \mathrm{~mm})$ and glucocorticoids $(42.2 \pm 8.4 \mathrm{~mm})(\mathrm{p}<0.05$ for both).

The duration of gout flares on different drugs was not significantly different and was on average 3 [1.5;4] days for the patients on NSAIDs, 5 [3;7] days for those on colchicine and $5[4 ; 6]$ days for the patients who received glucocorticoids.

The NSAID therapy was discontinued in two cases, in which the serum transaminase levels (AST, ALT) more than doubled; the colchicine therapy - because of development of diarrhea in two patients and of myopathy in one.

Conclusion: Efficacy of and tolerance to a three-month course of preventive therapy with NSAIDs and glucocorticoids in gout patients are comparable to that with colchicine. In case of development of gouty arthritis, preventive use 